ASEel SAMIR

Cairo University

(iD)

https://orcid.org/0000-0002-4741-1531

\section{Rabie Salama}

Luxor University

iD https://orcid.org/0000-0001-5711-8745

\title{
Struttura e narratore ne I Promessi Sposi di Alessandro Manzoni
}

\author{
Structure and narrator \\ in The Betrothed of Alessandro Manzoni
}

\begin{abstract}
In the early 19th century, the Italian literature did not have a mature novel, as is known today. The Italian novelist, Manzoni, and his masterpiece The Betrothed, set a solid basis for the contemporary Italian novel; thanks to its' narrative characteristics that helped the novelist in achieving different reformative goals, woven stupendously with fictional, historical and realistic threads.

The main purpose of this study is to apply an analytical and thematic approach on the structure and narrator of the novel. Furthermore, the research aims to distinguish the main artistic characteristics adopted from the European historical novel. The study then focuses on analyzing the function of the anonymous author's fictional frame and how it created a diversity in the narrative levels. The research also highlights the importance of the omniscient narrator, the strong relations between the narrator and the narratee, the different narrative perspectives, and finally the polyphony: techniques that enhanced the realistic dimension of the novel.
\end{abstract}

KEY WORDS: novel, manzoni, The Betrothed, structure, narrator

\section{Introduzione}

In Italia all'inizio dell'Ottocento, a differenza di altri paesi europei, non esisteva un romanzo maturo come lo conosciamo oggi nella sua forma complessa. Alessandro Manzoni, con la scelta di scrivere il suo romanzo storico I Promessi Sposi, ha lasciato un segno significativo nella letteratura italiana. Questa prestigiosa opera ha raccolto in sé numerose caratteristiche che l'hanno resa il centro d'analisi di importanti intellettuali. 
Una peculiarità di questo romanzo è la sua struttura che si è messa in sintonia con il genere dell'opera, e che è rimasta sempre fedele ai diversi livelli narrativi adottati dall'autore, portando così alla luce una costruzione omogenea.

La scelta stessa del genere del romanzo storico non è stata fatta a caso, ma è nata dal bisogno di rappresentare la realtà in maniera oggettiva, indagando nella storia motivi che si ripetono costantemente nelle varie epoche. Sapegno ha eseguito un'analisi sulle radici del romanzo e ha affermato che quella di Manzoni era un'idea di un romanzo composto da eventi inseriti in un'epoca storica e in un contesto sociale ben definiti (SAPEGNO, 1992: 100).

Questo capolavoro, nuovo all'Italia ottocentesca (cfr. ANGELINI, 1966: 10), ha ereditato la maggior parte delle sue caratteristiche strutturali da diverse fonti europee. Il romanzo manzoniano ha preso a modello i dibattiti degli intellettuali francesi e romantici, e ha adottato il modello romanzesco - se non la poetica della fantasia - dello scrittore Walter Scott e soprattutto del suo famoso romanzo Ivanhoe (cfr. PANEBIANCO, 1997: 140). Nonostante ciò, Manzoni è riuscito a combinare una propria poetica composta da elementi narrativi storici, intrecciati con una pura finzione creativa e basati sulla propria percezione del romanzo.

Questo articolo si prefiggerà di applicare un approccio analitico e critico allo studio delle tecniche strutturali dominanti in questo capolavoro, e avrà al contempo lo scopo di chiarire come Manzoni, nella veste di narratore e romanziere, è riuscito con bravura a tessere con fili variopinti una critica abile ed interpretativa, un impegno intellettuale riformatore e una realtà storica onnipresente non priva di una fantasia che si mescola fedelmente ad essa.

\section{La struttura generale del romanzo}

La scelta del genere narrativo ha avuto il suo eminente impatto sulla struttura del romanzo. L'autore, per raggiungere i suoi obiettivi, ha adottato naturalmente alcune caratteristiche che ricorrevano in genere nei romanzi storici (cfr. Cotroneo, 2009: 5), quali:

- Adoperare una struttura narrativa tradizionale caratterizzata da un equilibrio iniziale, rottura, peripezie e scioglimento.

- Ambientare le vicende in un periodo storico preciso che è il XVII secolo: Manzoni ha scelto un'epoca storica mai scelta in precedenza dagli scrittori italiani a lui contemporanei, i quali collocavano di solito le vicende narrative nel Medioevo.

- Ricostruire un contesto storico-sociale tramite la consultazione e lo studio di documenti storiografici appartenenti all'epoca, come le opere degli storici Ripamonti e Rivolta. 
- Inserire varie digressioni, come ad esempio le digressioni storiche sulle leggi seicentesche.

- Adottare la tecnica del narratore onnisciente.

- Operare una descrizione spazio-temporale precisa che si palesa nelle descrizioni dei luoghi e dell'atmosfera, dominanti sia in campagna che in città.

- Prestare grande attenzione alle relazioni tra i diversi strati sociali e alla classe borghese.

- Rappresentare figure storiche realmente esistite.

- Rivalutare la rappresentazione del brutto e non limitarla al bello per «dare una visione multiforme alla realtà» (COTRONEO, 2009: 5), come la rappresentazione degli episodi della peste.

Queste sono le caratteristiche proprie del romanzo storico; caratteristiche che l'autore ha adoperato, dando così alla luce una narrazione storica verosimile (cfr. GIRARDI, 1994: 13-15).

Ferroni ha affermato che Manzoni aveva l'esigenza di adottare la struttura del romanzo storico affinché «gli permettesse di allargare lo sguardo su un'intera società» (FERRONI, 1992: 639), dal momento che le strutture degli altri generi letterari si erano presentate come inadatte a realizzare tale scopo. Quindi le vicende narrate sono state inserite nel quadro tradizionale del romanzo storico europeo ottocentesco, e l'opera apparteneva ad «uno schema romanzesco tradizionale, quello dei due giovani innamorati la cui felicità è ostacolata da forze nemiche, ma che, dopo varie peripezie, riescono a ritrovarsi e a sposarsi» (642).

Vari critici hanno diviso la struttura dell'opera in sei «macrosequenze [...] più $\mathrm{o}$ meno lunghe e complesse, interamente suddivise in nuclei eterogenei» (MARChESE, 1986: 19), che in genere si possono distinguere in:

- Gli avvenimenti iniziali al paese natio (Capitoli: I-VIII).

- Le vicende di Monza (Capitoli: IX-X).

- Le insurrezioni milanesi e le vicende di Milano (Capitoli: XV-XVII).

- L'Innominato e Lucia, il rapimento e la libertà (Capitoli: XIX-XXIV).

- Le crisi: carestia, guerra e peste (Capitoli: XXVII-XXXV).

- Il finale della vicenda, il lieto fine e il ricongiungimento di Renzo e Lucia (Capitoli: XXXVI-XXXVIII) (cfr. Manzoni, 1999: 518).

Occorre specificare che la quarta macrosequenza, che occupa precisamente il capitolo XIX, si presenta nella metà esatta del romanzo, composto di trent'otto capitoli, e in cui appunto avviene il mutamento radicale che rovescia il resto del percorso narrativo, creando una divisione strutturale matematica ben studiata (cfr. Cotroneo, 2009: 14).

Tra le altre caratteristiche peculiari di questo romanzo, spiccano la creazione di una cornice introduttiva e l'inserimento di immagini figurative, grazie all'aiuto dei pittori Francesco Gonin e Luigi Sacchi. Questo inserimento ha avuto un valore molto apprezzabile in quanto ha dotato il romanzo di «eleganti incisioni» (FERRONI, 1992: 640) e di illustrazioni che hanno rappresentato la vi- 
sione dell'autore sugli avvenimenti più importanti, sull'aspetto e sulla figura dei personaggi. Manzoni si è anche occupato di fornire dettagli storici più specifici allegando all'opera l'appendice storica intitolata Storia della Colonna Infame ${ }^{1}$.

\section{Le diverse caratteristiche narrative}

\subsection{L'espediente dell'anonimo e i livelli narrativi}

Nella cornice introduttiva al romanzo, il narratore ha finto di aver trovato un manoscritto appartenente ad un autore anonimo risalente al Seicento. Questo manoscritto è stato apprezzato per la sua storia da Manzoni, il quale, di conseguenza, si è occupato di trasmetterlo ai lettori, facendo delle modifiche essenziali affinché questo manoscritto potesse essere presentato ai lettori ottocenteschi.

Il fatto di inserire nella cornice un fittizio scrittore e renderlo l'autentico autore degli eventi narrati, ha garantito ai lettori un senso d'originalità (cfr. RicCARDI, GIORGI, 2006: 579). Comunque, Manzoni ha rivelato ai lettori che questa cornice non era altro che mera invenzione, affermando quanto segue: «Ed ecco l'origine del presente libro, esposta con un'ingenuità pari all'importanza del libro medesimo» (Manzoni, 1962: 5). Lo scrittore con un tono ironico e tramite l'uso della parola ingenuità accennava esattamente al contrario intendendo una finzione.

Bussolaro ha analizzato il valore dell'espediente dell'anonimo, affermando che questo espediente si è integrato con il senso umoristico di Manzoni per realizzare una finalità linguistica ed ideologica:

Mentre in altri poeti e scrittori l'invenzione dell'anonimo non è se non un artificio, che sta in sé senza altre giustificazioni o finalità, nel Manzoni ha una sua profonda finalità, quella di ambientare inizialmente e di racchiudere $[\ldots]$ un giudizio che riguarda non soltanto la forma letteraria detestabile per la mancanza di sostanza, ma anche gli uomini che usarono tale forma espressiva in conformità ai loro modi di vivere e di considerarsi. L'anonimo, in seguito, verrà ripreso per confermare le istanze morali, umane e religiose.

Bussolaro, 2002: 33-34

\footnotetext{
${ }^{1}$ Si tratta di uno scritto storico elaborato da Manzoni e pubblicato ufficialmente nel 1842 in appendice al romanzo. L'autore, tramite questo scritto dedicato a documentare dati storici sulla peste di Milano e sugli accusati di diffondere il contagio, mirava a far coincidere il vero storico con quello morale, rivolgendo un'aspra critica all'intero sistema giudiziario del governo (cfr. Ferroni, 1992: 655).
} 
È necessario sottolineare alcuni esempi significativi in cui l'anonimo è stato citato esplicitamente nelle pagine del romanzo:

- Nelle informazioni biografiche fornite su padre Cristoforo: «Era figliuolo d'un mercante di $* * *$ 'questi asterischi vengon tutti dalla circospezione del mio anonimo'» (MANZONI, 1962: 88).

- Nella biografia della monaca di Monza: «accozzando questa qualsisia testimonianza con alcune altre indicazioni che l'anonimo lascia scappare sbadatamente qua e là» (245).

Ferroni ha ribadito l'importanza dell'inserimento dell'anonimo come espediente narrativo, in quanto:

dà luogo a una mescolanza tra passato storico e presente, tra oggettività storica e invenzione narrativa: chiamando in causa quell'antico testo come fonte del proprio discorso, l'autore può giocare a distinguere il proprio punto di vista da quello del fittizio originale.

FERRONI, 1992: 641

Questo processo ha preso così il via dall'incipit e ha continuato fino alla fine del romanzo, operando in tal modo su due piani narrativi «narratore di primo e di secondo grado» ("Analizzare il testo. Nozioni di narratologia", 2009: 12) quello dell'autore implicito e narratore ottocentesco manzoniano, e quello invece dell'anonimo seicentesco.

Insomma, i due piani narrativi si alternano per tutta la narrazione offrendo così una ricchezza ideologica propria sia di un'ideologia seicentesca che di un'altra ottocentesca. In effetti, viene constatato che il narratore manzoniano di primo grado «è un vero e proprio personaggio» (Cotroneo, 2009: 16), il quale stabilisce per tutta la narrazione «un gioco metanarrativo ironico e divertente» (16) con l'anonimo, ovvero con il narratore di secondo grado.

Di conseguenza, il narratore manzoniano si presenta con un atteggiamento variegato: paternalistico verso gli umili, ironico e sarcastico nella sua critica alla negatività della società e del potere, e compassionevole verso alcune figure femminili.

\subsection{Il narratore onnisciente}

Il narratore manzoniano è esterno, racconta $\mathrm{i}$ fatti in terza persona e narra una storia a cui non ha partecipato; quindi cerca di rielaborare la trama con un «processo di rielaborazione» ("Analizzare il testo. Nozioni di narratologia", 2009: 12) usando le proprie parole, oppure con un «processo di citazione» (12), cedendo la parola ad uno dei personaggi: 
Nei Promessi Sposi sono presenti entrambi i processi: la rielaborazione è costruita con l'espediente del manoscritto ritrovato "il narratore manzoniano rielabora la storia scritta dall'anonimo che ha rielaborato la storia sentita raccontare da Renzo", mentre il processo di citazione è messo in atto quando il narratore cede la parola ad un personaggio che narra una storia, come nel caso di fra Galdino².

“Analizzare il testo. Nozioni di narratologia", 2009: 12

Oltre a ciò, il narratore manzoniano risulta «eterodiegetico» (SEGRE, 2008: 25) ed onnisciente; sì che egli non appartiene ai personaggi della storia, ma è un narratore analista, sapiente che conosce tutti gli avvenimenti, i pensieri dei personaggi, le loro azioni e reazioni. Il narratore esprime così la sua valutazione nei confronti degli eventi e «si impone come giudice» (20), esibendo i propri giudizi oppure i giudizi presunti di chi avrebbe commentato gli stessi eventi, ed offre insomma diverse interpretazioni e riflessioni.

L'onniscienza del narratore manzoniano gode di un carattere assoluto «che pare avere l'ampiezza di uno sguardo divino» (Ferroni, 1992: 648). Tuttavia, esistono alcune novità introdotte da Manzoni, che lo distinguono dalla narrativa tradizionale precedente, mediante l'inserimento di:

vari schermi, sfasature, complicazioni, che da una parte arricchiscono il racconto di effetti concreti di realtà e di verità, dall'altra indicano l'inevitabile differenza esistente tra il significato più autentico degli eventi e l'interpretazione che lo stesso narratore ne può dare.

FERRONI, 1992: 648

\subsection{I diversi legami del narratore onnisciente con la materia narrativa}

Il narratore onnisciente si lega alla materia narrativa facendo prevalere la funzione sia diegetica sia mimetica (cfr. SEGRE, 2008: 16-22), alternandole per tutte le pagine del romanzo. Da una parte, il narratore si manifesta nella forma diegetica nelle descrizioni, nei commenti, nei riassunti e nei resoconti. Spicca come esempio della forma narrativa diegetica: «Risparmio al lettore i lamenti, le condoglianze, le accuse, le difese, i "voi sola potete aver parlato", e i "non ho parlato", tutti i pasticci in somma di quel colloquio» (MANZONI, 1962: 54).

Dall'altra, nella forma mimetica, nei dialoghi e nei soliloqui, il narratore ha cercato di affidare la parola ai suoi personaggi, senza smettere mai di commentare o di interpretare ciò che accadeva. Perciò, occorre sottolineare che Manzo-

${ }^{2}$ Si tratta qui della storia del miracolo delle noci raccontata da Fra Galdino con un processo di citazione costituendo anche una modalità metanarrativa (cfr. MANzoni, 1962: 79-80). 
ni, nella veste di narratore onnisciente, ha ceduto parzialmente, e non completamente, la parola ai suoi personaggi. Si nota come esempio di questo miscuglio narrativo diegetico-mimetico:

- Sentite, Lucia, sentite! - disse Renzo, senza però accostarsele di più.

- No, no; andate per carità!

- Sentite: il padre Cristoforo.... [...] - Gli ho parlato poco fa; sono stato un pezzo con lui: e un religioso della sua qualità, mi pare....

- È qui! per assistere i poveri appestati, sicuro. Ma lui? L'ha avuta la peste?

- Ah Lucia! ho paura, ho paura pur troppo.... - e mentre Renzo esitava così a proferir la parola dolorosa per lui, e che doveva esserlo tanto a Lucia, questa s'era staccata di nuovo dal lettuccio, e si ravvicinava a lui: - ho paura che l'abbia adesso!

MANZONI, 1962: 950

Un'altra caratteristica narrativa ricorrente è il ricorso del narratore onnisciente all'uso della metalessi ("Analizzare il testo. Nozioni di narratologia", 2009: 13), grazie alla quale la voce del narratore può intervenire direttamente nella narrazione «a sottolineare la sua funzione di regia» (MARCHESE, 1986: 19).

Mediante la metalessi il narratore di primo grado interviene continuamente nella storia facendo ricordare costantemente la propria presenza e dialogando pure con il narratario ("Analizzare il testo. Nozioni di narratologia", 2009: 10). Intanto, il rapporto tra il narratario e il narratore si basa su continue intrusioni da parte di quest'ultimo. Il narratore si rivolge a dei lettori impliciti o meglio narratari, dal momento che sono stati precisati e citati esplicitamente sin dall'esordio del romanzo: «Pensino ora i miei venticinque lettori che impressione dovesse fare sull'animo del poveretto, quello che s'è raccontato» (MANZONI, 1962: 31).

Questi narratari godevano nella mente di Manzoni di caratteristiche più o meno constatabili, quali: «appartenenza al ceto medio, cultura medio-alta» ("Analizzare il testo. Nozioni di narratologia", 2009: 10).

La scelta del lettore non era casuale, infatti, questo romanzo storico era rivolto principalmente alla classe borghese, ma al contempo, per finalità divulgative e culturali, era anche indirizzato ad altri strati sociali.

Così, il ruolo del narratario è rimasto attivo in quanto veniva solitamente chiamato dal narratore con «ragguagli narrativi ed extranarrativi, [...] appelli al lettore, $[. .$.$] metalessi metanarrative» (COTRONEO, 2009: 16), come verrà consta-$ tato negli esempi seguenti:

- Ragguagli narrativi ed extranarrativi per informare il lettore di ulteriori dati:

«Don Abbondio "il lettore se n'è già avveduto" non era nato con un cuor di leone » (MANZONI, 1962: 25). 
«Il lettore sa che in quell'anno si combatteva per la successione al ducato di Mantova » (130).

- Appelli al narratario con lo scopo di invitarlo a partecipare alla storia narrata, a dare dei giudizi o persino a fare delle inferenze:

"Questioni importanti; ma che il lettore risolverà da sè, se ne ha voglia. Noi non intendiamo di dar giudizi: ci basta d'aver dei fatti da raccontare» (148).

- Appelli al narratario per farlo riflettere su un certo dato o fatto:

"Questo termine è rimasto e vive tuttavia [...] nel dialetto: e non ci sarà forse nessuno de' nostri lettori milanesi, che non si rammenti d'aver sentito, [...], dir di lui: è un ciuffo» (73).

- Metalessi metanarrative per riflettere sui modi del raccontare gli eventi:

«Intorno a questo personaggio bisogna assolutamente che noi spendiamo quattro parole: chi $[. .$.$] avesse però voglia d'andare avanti nella storia, salti$ addirittura al capitolo seguente» (732).

Squarotti ha analizzato il motivo per cui il narratore manzoniano interveniva perpetuatamene nella narrazione, negando il carattere oratorio attribuito a Manzoni dalla critica precedente e affermando che essa non era altro che «un modo tipico di conoscere la realtà secondo una straordinaria duplicità di prospettive » (SQuarotti, 2001: 522). Quindi, Manzoni aveva in mente lo scopo di riflettere la propria prospettiva: «Nulla è lasciato, dal Manzoni, nell'indeterminazione, nell'indifferenza, nella libertà assoluta dell'oggettività» (522).

\subsection{La prospettiva narrativa}

Per quanto riguarda il concetto della prospettiva narrativa nel romanzo, ovvero il punto di vista secondo cui la narrazione si svolge, si nota che il narratore manzoniano ha adottato diversi tipi di focalizzazione (cfr. MARCHI, 1997: $25-28)$.

La «focalizzazione zero» (Segre, 2008: 27) è quella dominante nella narrazione, dato che il rapporto tra la quantità di informazioni che connette il narratore ai suoi personaggi è di tipo «Narratore > Personaggio» (27), ed è un risultato naturale dell'onniscienza narrativa, di colui che sa esprimersi più di quanto non sappiano fare gli stessi personaggi e non si lascia dominare completamente dalla loro prospettiva.

Si potrebbe, inoltre, constatare una «focalizzazione interna fissa» (27) negli episodi ironici di Don Abbondio ad esempio, in cui il narratore espone i fatti secondo la prospettiva del curato, e non secondo la propria prospettiva.

L'uso di questo tipo di focalizzazione ha creato uno «straniamento» ("Analizzare il testo. Nozioni di narratologia", 2009: 13), e, conseguentemente, ha suscitato un effetto di «scarto ironico» (13) ai lettori, dal momento che il narratore ha esposto i fatti secondo la «mentalità anomala» (13) del curato: 
la sua mentalità è distante da quella del lettore medio e sicuramente da quella dell'autore. Così ogni volta che Don Abbondio appare in scena la denuncia è sottintesa, ma il lettore percepisce l'ironia dell'autore ed è chiamato a giudicare autonomamente il personaggio.

Cotroneo, 2009: 17

Eppure, esiste anche la «focalizzazione interna multipla» (SEGRE, 2008: 27) che emerge, ad esempio, nella notte degli imbrogli nel capitolo VIII, focalizzata e vissuta tramite gli occhi di più personaggi (cfr. ManzonI, 1962: 198-211).

Peraltro, l'uso della «focalizzazione esterna» (SEgRe, 2008: 27) ricorre di rado e, quando avviene, si connette con le focalizzazioni già citate. Tale uso può essere constatato nell'episodio in cui, per la prima volta, Renzo arriva a Milano, e in cui il narratore si limita a descrivere il caos della città tramite la prospettiva del personaggio, senza fornire nessun'informazione al riguardo.

Questo tipo di focalizzazione ha anche creato un senso di straniamento dando vita ad uno scarto ironico: poiché nella mentalità anomala di Renzo, montanara e ignara della grande città, Milano appariva come «il paese di cuccagna» (MANZONI, 1962: 320), il lettore si trova costretto ad indovinarne e giudicarne i motivi senza interventi diretti da parte del narratore.

L'uso delle focalizzazioni e l'adozione di diversi e insoliti punti di vista hanno permesso al narratore «di denunciare situazioni, scelte, idee e atteggiamenti assurdi, senza intervenire direttamente con giudizi e condanne esplicite» (Cotroneo, 2009: 17).

\subsection{La polifonia}

Grazie all'adozione di diverse focalizzazioni e prospettive è venuta alla luce una certa polifonia, ovvero una pluralità di voci, come hanno sostenuto alcuni critici, tra cui spicca Angelo Marchese. Sebbene il narratore risulti il regista delle numerose voci dei personaggi, egli esprime le loro voci e prospettive più personali. Come accennato in precedenza, le varie voci dei personaggi non appaiono solamente tramite una narrazione diegetica, ma compaiono anche attraverso la mimesi.

L'esempio più significativo della polifonia va riscontrato nelle vicende delle insurrezioni a Milano, in cui esiste una forte pluralità di voci. L'uso della polifonia, che ha avuto la funzione di rispecchiare il caos creato dai tumulti, qui ha fatto nascere una certa confusione:

- Ora è scoperta, - gridava uno, - l'impostura infame di que' birboni, che dicevano che non c'era nè pane, né farina, nè grano [...] - Vi dico io che tutto questo non serve a nulla, - diceva un altro: - è un buco nell'acqua; anzi sarà peggio, se non si fa una buona giustizia. 
L'uso polifonico nella narrazione è stato in grado di offrire una complessità di punti di vista che hanno arricchito la materia narrativa: la polifonia ha conferito alla narrazione un forte senso di realismo, proponendo una molteplicità di prospettive che esistono solamente nella realtà (Bologna, RocCHI, 2010: 514).

Marchese, nella sua analisi della polifonia presente nella narrazione manzoniana, si è basato sulla teoria polifonica elaborata da Michail Bachtin, applicando la concezione del critico russo per ciò che concerne le caratteristiche principali di cui un autore di romanzo polifonico, come Manzoni, è solitamente dotato.

In effetti, Manzoni è apparso come un autore in grado di raccogliere in sé le coscienze degli altri, senza racchiudersi in sé e senza cancellare la propria coscienza. La polifonia del romanzo manzoniano può essere constatata in vari episodi, tra cui spicca il famoso finale del romanzo in cui vengono rappresentati l'essenza e il significato dietro le traversie che i protagonisti hanno sperimentato. Difatti, tale finale è stato inscenato tramite le prospettive e le voci dei due protagonisti.

Per di più, Marchese ha eseguito un'analisi in cui ha collegato la tecnica della polifonia a quella del narratore onnisciente, deducendo che Manzoni nella veste di autore implicito:

distingue nettamente le parti dei personaggi, del narratore e del lettore, esigendo da quest'ultimo, oltre a un massimo di consenso e di sintonia spirituale, un impegno critico nella salvaguardia della propria autonomia di giudizio, quasi fosse una "mente estrinseca" che non si indentifica mai con la favola.

Marchese, 1991: 186

Come risultato, Marchese ha affermato che i personaggi dell'opera hanno una certa autonomia, nonostante la presenza di un narratore onnisciente che concede il punto di vista e la parola ai suoi personaggi e:

ne valorizza l'indipendenza assiologica e rende possibile, nella caleidoscopica diversità delle prospettive a confronto, il manifestarsi di quella polifonia o concertazione di voci che concorre, sotto un'abile e discreta direzione, alla genesi corale del messaggio artistico.

Di conseguenza, viene rifiutato il carattere di onniscienza divina, assoluta e totalizzante affidato al narratore manzoniano dalla critica, e viene attribuito a quest'onniscienza un carattere piuttosto condizionato, tecnico, meditativo e morale proprio di un autore storico e intellettuale. 


\section{Conclusione}

La scelta de I Promessi Sposi di Manzoni come il romanzo su cui basare questa ricerca è stata presa con molta cura, dal momento che esso, come è stato messo in risalto da vari critici, è un capolavoro che ha dato vita alla forma del romanzo nel suo senso moderno in Italia (SAPEGNO, 1965: 852-858). In effetti, il romanzo storico manzoniano risulta un'opera molto ricca in sé, sia sul piano strutturale che su quello narrativo.

La struttura tradizionale dell'opera, nonostante fosse ispirata da altre fonti europee, ha comunque avuto la sua originalità ed è stata contraddistinta da una divisione precisa in trent'otto capitoli, dando così alla luce sei macrosequen$z e$ principali che hanno raggruppato i principali avvenimenti della trama. Oltre a ciò, la cornice introduttiva e l'espediente dell'anonimo hanno arricchito non solo la struttura, ma anche i livelli narrativi. Si è così creato un gioco metanarrativo dilettevole secondo cui la struttura viene inserita in una cornice generale, cosa che ha permesso al narratore manzoniano di conferire al testo una variegatura e alternare i livelli narrativi tra un passato seicentesco e un presente ottocentesco, tra realismo storico e finzione narrativa.

Questa narrazione storica aveva naturalmente il bisogno di un narratore onnisciente ed eterodiegetico capace di ben collegarsi alla sua materia narrativa sia con processi di elaborazione che con processi di citazione, aggiungendo con bravura alla narrazione un miscuglio diegetico-mimetico.

Tuttavia, la prospettiva narrativa non si è limitata ad un ritmo monotono, anzi, il narratore manzoniano ha adottato vari tipi di focalizzazione, da quella zero e quella interna fissa a quella interna multipla ed esterna. Grazie a tutta questa varietà di prospettive è nata nel romanzo una polifonia che a sua volta ha conferito alla materia narrativa caratteristiche realistiche.

Per completare questo mosaico realistico, nella veste di narratore, Manzoni ha desiderato coinvolgere i suoi lettori utilizzando la tecnica della metalessi, la quale ha stretto profondi legami tra il narratore e il narratario. Quest'ultimo viene invitato a partecipare alla narrazione attraverso ragguagli narrativi ed extranarrativi, appelli e metalessi metanarrative.

Tutti questi sforzi e tecniche del narrare corrispondono principalmente alla poetica manzoniana, secondo cui l'opera letteraria è fondata su «l'utile per scopo, il vero per soggetto e l'interesse per mezzo» (BologNA, Rocchi, 2010: 459), e la vera essenza del romanzo è basata appunto su un impegno serio da parte di Manzoni a illuminare la mentalità dei suoi lettori. Egli, quale impegnato interprete della storia e della realtà dell'Italia a lui contemporanea, ha fatto propria la missione di divulgare la cultura e la letteratura, diffondendo le sue idee presso i più ampi strati sociali. 


\section{Bibliografia}

"Analizzare il testo. Nozioni di narratologia", 2009, in: I Promessi Sposi. Laboratorio. Milano, Editore Ulrico Hoepli.

Angelini, Cesare, 1966: Capitoli sul Manzoni vecchi e nuovi. Verona, Arnoldo Mondadori Editore.

Bologna, Corrado; Rocchi, Paola, 2010: Rosa fresca aulentissima. Neoclassicismo e Romanticismo. Vol. 4. Torino, Loescher.

Bussolaro, Domenico, 2002: Temi svolti su "I Promessi Sposi”. Calvizzano (NA), Il Girasole.

Cotroneo, Dorotea, 2009: I Promessi Sposi. Alessandro Manzoni. Milano, Ulrico Hoepli Editore.

Ferroni, Giulio, 1992: Profilo storico della letteratura italiana. Vol. 2. Torino, Einaudi Scuola. Girard, Enzo Noè, 1994: Struttura e personaggi dei "Promessi Sposi". Milano, Jaca Book.

Manzoni, Alessandro, 1962: I Promessi Sposi. A cura di Enrico Bianchi. Firenze, Le Monnier.

Manzoni, Alessandro, 1999: I Promessi Sposi. A cura di Maria A. Garavaglia. Milano, Mursia Scuola.

MarcheSE, Angelo, 1986: Come sono fatti "I Promessi Sposi". Guida narratologica al romanzo. Milano, Arnoldo Mondadori Editore.

Marchese, Angelo, 1991: "La polifonia nei Promessi Sposi". In: AA.VV.: Atti del XIV Congresso Nazionale di Studi Manzoniani. A 150 anni dalla edizione 1840 dei "Promessi Sposi”. T. 1. Milano, Centro Nazionale di Studi Manzoniani. Casa del Manzoni, pp. 185- 201.

Marchi, Alessandro, 1997: Alla scoperta del testo narrativo. Percorsi di analisi e antologia di testi. Torino, Petrini editore.

Panebianco, Beatrice, 1997: Ritratti d'autore. Bologna, Zanichelli.

Riccardi, Cecilia; Giorgi, Tiziana, 2006: Antologia Europea. Futura Narrazioni. Bergamo, Paravia Bruno Mondadori.

SAPegno, Natalino, 1965: "I Promessi Sposi e il loro significato nella letteratura italiana". In: Walter Binni, Riccardo Scrivano: Antologia della critica letteraria. Milano, Principato, pp. 852-858.

SAPEGnO, Natalino, 1992: Ritratto di Manzoni e altri saggi. Bari, Laterza.

Segre, Cesare, 2008: Avviamento all'analisi del testo letterario. Torino, Biblioteca.

SQuarotti, Giorgio Barberi, 2001: "La composizione metafisica nello stile dei Promessi Sposi". In: Guido Baldi, Silvia Giusso, Mario Razetti, Giuseppe Zaccaria: Dal testo alla storia. Dalla storia al testo. Dal Neoclassicismo al Verismo. Vol. 3/1². Milano, Paravia Bruno Mondadori, pp. 522.

\section{Note biobibliografiche}

Aseel Samir - Assistente docente e ricercatrice di lingua e letteratura italiana presso la Facoltà di Lettere - Università del Cairo, con specializzazione in letteratura italiana moderna. Ha ottenuto il Master di Ricerca, con il massimo dei voti, dalla Facoltà di Al-Alsun - Università di Ain Shams, e si è laureata a pieni voti in studi d'Italianistica dall'Università del Cairo.

aseelareeg92@gmail.com 
Rabie Salama - Professore Ordinario di letteratura italiana e traduzione presso la Facoltà di Al-Alsun - Università di Ain Shams, e Preside della Facoltà di Al-Alsun - Università di Luxor. Ha condotto vari studi riguardanti la poesia, il romanzo e il teatro nelle diverse epoche della letteratura italiana, dall'età medievale fino alla modernità e l'età contemporanea. Ha inoltre pubblicato varie ricerche riguardanti la letteratura comparata e gli studi della traduzione (Italiano-Arabo).

prof.rabiesalama@yahoo.com 